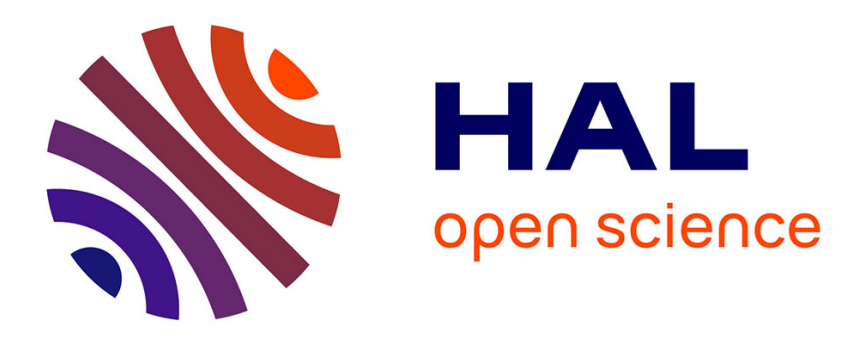

\title{
Time-free Description of Motion in Dynamic Systems From Momentum to Entanglement
}

\author{
Joseph J Jean-Claude
}

\section{To cite this version:}

Joseph J Jean-Claude. Time-free Description of Motion in Dynamic Systems From Momentum to Entanglement. 2017. hal-01965924

\section{HAL Id: hal-01965924 \\ https://hal.science/hal-01965924}

Submitted on 27 Dec 2018

HAL is a multi-disciplinary open access archive for the deposit and dissemination of scientific research documents, whether they are published or not. The documents may come from teaching and research institutions in France or abroad, or from public or private research centers.
L'archive ouverte pluridisciplinaire HAL, est destinée au dépôt et à la diffusion de documents scientifiques de niveau recherche, publiés ou non, émanant des établissements d'enseignement et de recherche français ou étrangers, des laboratoires publics ou privés. 


\title{
Time-free Description of Motion in Dynamic Systems From Momentum to Entanglement
}

\author{
Joseph J. JEAN-CLAUDE \\ May 19, 2017, (C) Copyright \\ quantogeometry@gmail.com
}

\begin{abstract}
Time is in serious trouble nowadays in physics. From every corner skepticism is mounting up and some of those physicists who would have put their hands in the fire in yesteryears in support of its ubiquity are nowadays becoming somewhat more receptive to the idea that it might be unreal or at least real only as a local variable. In the Quanto-Geometric Theory we have proposed new physics and novel solutions in response to the many conundrums facing the field which radically proscribe time in the metric of the physical realms. What is difficult to construct in a program of timeless physics is an interpretation of the evolution of time-independent dynamic systems at all scales. While we already know what that looks like for the quantum world with E. Scrodinger's Quantum Mechanics, albeit to reserved satisfaction of many who feel that the theory is incomplete, the endeavor of a granular dynamic description of time-independent physics in the macrocosm has probably yet to be achieved. We make this objective the subject matter of this thesis, in the understanding that such a program cannot be thoroughly realized in the cast of one single paper. Nonetheless in this proposal we undertake to substantially muster up a time-free description of motion in dynamic systems from momentum to entanglement, in physics at all scales.
\end{abstract}

Keywords: mathematical physics, theoretical physics, quanto-geometry, quanto-geometric theory, quantum mechanics, relativity, unified quantum relativistic background, quanto-geometric operators, quantogeometric tensors, shells of matter, time-free motion, time-independent dynamic systems, time-free evolution of dynamic systems, timelessness, timeless physics, omniverse, World Plane, shells or matter, Schrodinger's cat, quantum entanglement

\section{Introduction}

In his continual search for a global description of the 3dimensional World, John Wheeler had advanced "that time may not be a fundamental aspect of reality, but [that] this only happens on extremely small distance scales" [8]. More audaciously, we contend that time physically participate in no aspect of reality at all. In the following we address a factual numeric description of the evolution of dynamics systems on a time-independent basis where it has not been undertaken before. We further offer a rational explanation for quantum entanglement that is bereft of the outright absurdity of retrocausation.

\section{Inappropriate Role of Time in the Descrip- tion of Motion}

When we examine motion and time alone, one quickly and routinely falls under the impression that any value of motion and any chronological value of time are and must be possible. All classical or neo-classical view of physics, which is also the view of the larger public, belongs to this school of thought. We ordinarily further assume that all dynamic systems must concord with this determination, independent of the objects in action or interaction. Despite the fact that Einstein's Theories of Relativity have shown the peremptory limit of motion in the 3-D World Plane, we have yet to understand the implications of the limit of motion on time and continue to visualize time as this ad infinitum continuum.

Lorentz invariance ultimately teaches that nothing can be accelerated to the speed of light with respect to the observer. One way to make sense of the situation of an object in acceleration is to approach it from the point of view of change in momentum or change in energy. Considering the change in momentum $(d p / d t)$ as the object is being accelerated to $c$, we commonly say that it would require an infinite amount of time to accelerate the object to $c$, since:

$$
\frac{d p}{d t}=m \cdot \frac{d v}{d t},
$$

where $p$ is the momentum, $v$ the speed or velocity and $m$ the mass of the object.

For the derivative above to describe an accelerating situation that would bring the speed of the object to $c$, we must read that as $d t \rightarrow 0$, $d v$, the incremental of velocity between two successive points, must become larger, which makes for a steep variational slope. In other words, there must be larger increments of speed for smaller increments of time for acceleration to occur toward reaching $c$. But we know that the instantaneous speed (most affine incremental) experienced by the object can only reach the neighborhood of $c$ from the left 
side but not $c$. So therefore there is a limit on $d v / d t$. Counterintuitively, the limit on $v$ should not be applied to $d v$ in terms of displacement $\Delta x$ (given that $v_{a v}=\Delta x / \Delta t$ ), because there is nothing in isotropic space to intrinsically obstruct objects's displacements and prevent any desirable value of $\Delta x$. Consequently the limit on $v$ must be reported to $d t$, which in this context becomes the unit of the scale of time as a human creation, in terms of a lower limit. The general consensus conclusion drawn from this situation is that it would take an infinite amount of time to accelerate an object to the speed of light. Instead the conclusion should be that $c$ is the limit of time, since it imposes a lower limit on the unit of time. The smallest possible bit of time $\tau$ is that one which would bring an object from speed $v<c$ to $\sim c$. No smaller bit of time $\tau<\tau$ is possible because it would bring the objects's speed to $c$ or over $c$. However, the limit imposed on time by $c$ is counter-intuitive because it gives time a life as a quantum or discrete packet while time in our minds is a variable submissive to gradation by any quantity $a$ such that $a \mathcal{E} \mathbb{R}^{+}$, the realm of positive real numbers. In chronology, we do not add time by discrete bundles but by any finite numeric quantity we please according to the cardinality property of the real number realm. We chose the second to be the unit of time, in the understanding that it could have been any numeric value of duration of our election and that any total number of seconds is possible in chronology, as much as any fractional value of the same. In any case, the standard second is certainly not the smallest possible gradient of time as implied by its relationship with $c$.

The idea of a Planck time, responding to a search for a natural unit of time, circumvents the logic that relates time to $c$ by simply projecting an expectation value of the unit of time from the value of $c$, whilst $c$ is already expressed in unit of time. Its formula:

$$
t_{P}=\sqrt{\frac{\hbar G}{c^{5}}}=5.39116(13) \cdot 10^{-44} \mathrm{~s}
$$

where $\hbar$ is the reduced Planck constant ( $h$ instead of $\hbar$ is sometimes used in this definition, but most commonly $\hbar$ ), $G$ the gravitational constant, $c$ the speed of light in a vacuum and $s$ the SI unit of time, the second. It is the most perfect vicious circle.

Deriving from a clear vicious circle, the Planck time notion is not and cannot be a legitimate projection. Thus the idea of a Planck unit of time is meaningless. Not in vain has it not been found in nature. Obviously if a natural unit of time cannot be found, time must be demoted from its dimensional stature, officially declared a human standard destined to disap- pear and a new time-free description of physics altogether summarily sought, on the basis of a dimensional time-free metric.

Note that had we conducted the analysis by starting with Energy instead of momentum, the conclusion we were driven to would have been exactly the same.

Finally, there is indeed a paradox of note in our conception of time, in that we attribute a discrete bundle unit to time, whatever its duration value is, while we are ready to accept that a chronological segment of time can take any finite value whatsoever. In other words, we do not have indeed a physical metric of time. In our minds, time is as loose as anything can be. At a minimum, we manage time as a mathematical entity incarnated in the positive real number domain. That assessment has prompted some to argue that time is real because it behaves as mathematical ordinals, an argument often coming from dissident physicists adverse to time and even from A. Einstein himself who wrote:

"Time has no independent existence apart from the order of events by which we measure it."

In fact we do not measure time by the order of events as he asserts but we observe events and rationalize their sequential order by measuring or assessing their flow in time. So therefore, what he meant to say, in our estimation, is that we warrant time independent reality or legitimacy only on the basis of its equivalence with mathematical ordinalization. In point of fact, assimilating time to mathematical ordinals makes it an abstraction to the same extent as mathematical entities themselves and poses the problem of whether or not it is adequate to use mathematics to model time as a putative physical variable, such as done in all proposed metrics in all-time physics. The intrinsic incorporation of time to mathematics has presumably been a late effort by modern-day physicists who cannot accept the world without time and developed their own time-based mathematics, i.e. ergodic theory, even though some of these physicists were also genuine mathematicians. The mathematical-physics proposition in science, shall we say, quickly becomes a tricky proposition if its main attempt is to blur the lines between physics and mathematics instead of developing the lines of connection between the abstract model that mathematics indeed represents and the physical reality purported to be the main object of physics, which mathematics should solely aim to model. 


\section{Why Acceleration is Equivalent to Inertial Motion}

Since the $6^{\text {th }}$ century of our era, John Philoponous knew that two balls of very different weights will fall at approximately the same speed. In 1586, Simon Stevin dropped two lead balls of very different masses off the Delft church tower, for them to reach the ground with no detectable difference in arrival time as recorded. Those results are not necessarily intuitive insofar as the mass quantities involved. It so occurs that objects in free fall, or in accelerated motion, do not register an experience of being accelerated, in this case toward the center of Earth, but rather weightlessness and no acceleration at all. If an accelerometer is tied to such falling object, the device does not register any acceleration at all, so demonstrating that there is veritably none.

Examining this situation in the context of developing a theory of gravity, A. Einstein concluded that accelerated motion and inertial motion were physically equivalent to one another. The question is seldom posed as to what the reason behind the equivalence principle could be in physical terms as it relates to the types of motion. One may argue that Einstein sought an answer to this question by establishing the theory of space or spacetime as the mediator of gravitational interaction. A counter-argument is that a theory of the origin of motion is a better answer to the question and that a gravitational theory of motion is an appropriate answer only if one assumes that gravitation is a primitive of motion in general. Furthermore, Newton's gravitational description, which views gravitation as an attraction Force exerted on a body, expressed by the inverse square law, remains a legitimate and numerically accurate answer to this question. To this day we do not know which one of the two approaches best describe gravity [11].

Our aim in this paper is to attempt a description of the very intimate origin of motion. While General Relativity gives a view of motion in the context of gravitation, it does not quite explain the origin of motion. It builds on the observational fact that accelerated motion is equivalent to inertial motion and essentially offers the proposition of the geometric structure of spacetime as the mediator of gravitational motion. Let us start by comparing inertial motion to accelerated motion. Conceived as a displacement in time, pure inertial motion is described by the derivative in time:

$$
v=\frac{d x}{d t}
$$

Whereas pure acceleration is described as the variation of speed in time:

$$
a=\frac{d v}{d t}
$$

One way of looking at acceleration is to view it as a step function in time, whereby at each time increment, the speed jumps to a higher value.

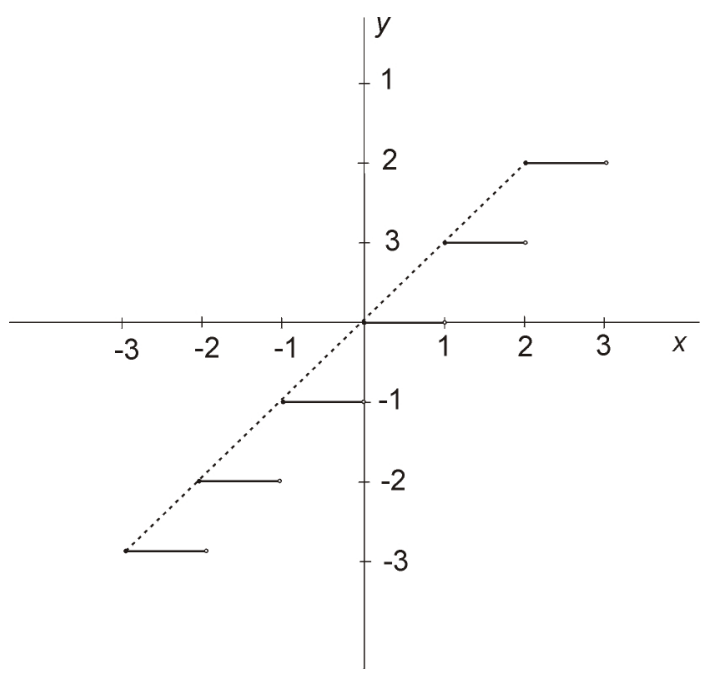

Fig. 1 The greatest integer function as a step function approach to acceleration

How long an instantaneous value of the speed at one time jump remains constant during the open time segment or time interval is immaterial to the overall step behavior of the motion, as long as the time interval remains constant to make for a motion with constant acceleration. The greatest integer function $(f(x)=[x])$ is a very good model of this vision of acceleration (Fig.1). The most irreducible of such step functions is the one with the most affine time intervals (steps of the stairs), while the jumps in time are to be almost perfectly steep. The function is obviously discontinuous at those step or jump points in time. For a function with the most affine possible steps, one can see that it reduces to the linear function obtained by joining the step points as shown in Fig.1. That linear function is a pure expression of speed or inertial motion.

Therefore at the limit when $d t \rightarrow 0, a$ becomes:

$$
a \equiv k \cdot v
$$

where $k$ is a slope factor. It is important to recognize that in the neighborhood of any considered step point where $d t \rightarrow 0$ is evaluated the function no longer behaves in time because of its discontinuity at these time points. Hence we must forcibly conclude that if accelerated motion ought to be equated to inertial motion, this transformation is not and cannot be realized in time, but only within the dimension of displacements, within the space dimension, that is. 


\section{To Describe Motion Exclusively as a Func- tion of Space}

We have discussed above the reasons why, should $c$ be the limit of motion, it consequently sets a lower end limit to the scale of time. Let us now examine what happens at the upper end of the scale. When we express motion in $\mathrm{m} / \mathrm{s}$ with $c$ as the scale encompassing the gradients of $\mathrm{m} / \mathrm{s}$, we are settling for tallies or units of $\mathrm{m} / \mathrm{s}$ from 0 to $c$ where $c$ is the maximum value of $\mathrm{m} / \mathrm{s}$.

We know what $c$ looks like as a pure scalar and not a physical unit in time: $0.299796065 \sigma^{-1}$, a number derived from plenary mathematical construction in [1] or [2]. In an attempt to give physical meaning to $\sigma$ in that construction, we compare $\sigma$ to $\mathrm{m} / \mathrm{s}$ by first looking at how many dimensions there can be in $\sigma$. In the Quanto-Geometric 2-space metric, it is a one-dimensional parameter. In all, we have the choice of either giving it the value of time $(\mathrm{s})$ or the value of space $(\mathrm{m})$. If we give it a value of choice from the metric of space, we obtain the quantity $\mathbf{2 9 9 , 7 9 2 , 4 5 8}$ that we well know, which is a quantity of motion, as demonstrated in [1] [2]. But if we wanted to give it a value from the metric of time, we cannot find a value from such metric to assign to it, because there is no natural time metric. A value dictated by a natural time metric would be the only acceptable one in this case. So we must conclude from this lack that the quantity 0.299796065 $\sigma^{-1}$ does not recognize time or is not a fit to time. It must strictly be a descriptor for motion exclusively in terms of space.

Because all motion must have a geometric descriptor, and since every basis manifold is associated with a symmetry modulus that is a scalar, every motion becomes innately and discretely quantifiable, according to the metric germane to its associated eigenform. This firmly holds despite the fact that the continuum nature of space makes it generically subject to any metric measure. In its realization, however, physical space becomes differentiable or distinguishable without quantization. This differential order is motion or the origin of motion.

One cannot imagine motion without a constant, fixed or running, even though a running constant is no longer quite a constant. For a displacement to occur, the end point must be related to a fixed start point or a moving start point at a different lower or higher rate of motion, not only in representation but principally physically. In this hierarchy, displacement from a fixed point becomes a particular case of the general situation of a running start point. Therefore if we have to let motion be true to itself, which is what occurs at the asymptotic level of quanto-geometric distribution or in all authentic linear metrics, motion must start from infinite rate, which is when there is no incremental at the denominator of the fractional rate, and pursue by degeneration as the incremental at the denominator starts taking numeric values and trending to increasingly larger values. What does this mean? Squarely the following:

1. When there is no incremental at the denominator of the generically descriptive fractional rate, then there is no rate and the value of the displacement is any infinitely large value one may care to imagine.

2. Subsequently when there is an infinitesimally small value at the denominator then the rate is at a very large value but a measurable or countable one.

3. As the incremental value at the denominator increases, the rate of motion diminishes accordingly. Should this value become infinitely large then the rate of motion drops to the almost standstill value of zero.

This flow rate is not $d x / d t$ which we are all so very familiar with but $d r / d s^{l}$, the rate at which the very fabric or tapestry of space defoliates or un-folds by itself. It mediates what we have otherwise called elsewhere the propagation of space. In the hierarchy of endogenous space motion or space momentum, the arrow of space is from very large to very small, whereas in our intuitive metric of time, as consecrated in all postulated time-related metric thus far (i.e. FLRW metric), the flow (rate) of time evolves from smaller to larger. There is no wondering what the cause of this motion is, because the cause belongs in the very definition of the spatial spread: in QuantoGeometric Theory space is ontologically kinetic, since it is orthogonal to the scalar [1], its intrinsically invariant and static ontological partner. There is no wondering what it is that space leaves behind in its perpetual defoliation, because there is only left the back scene of the infinites behind. Both from the tensor and the propagation descriptors, space is totalitarian: there can never be a gap in space since a gap is a spread of void, a space spread necessarily.

What we have called elsewhere the $s$-line or the superscale imposed on the metric of 2-space [1] [2] determines the values or gradients of motion accessible to space, firstly on the map of the Omniverse or the Mappa Mundi, and secondarily by surrogation in the development of the World Planes.

We reproduce here once again the super-imposed scale over the 2-space metric:

$$
\begin{aligned}
& \text { for } n=1, \mathrm{~s}_{1}=0, \text { or to be exact } s_{1} \rightarrow 0^{+} \\
& { }^{1} \text { Note that the flow rate is in fact: }-\left|\frac{d r}{d s}\right| \text {, as later explained. }
\end{aligned}
$$




$$
\begin{aligned}
& \text { for } n=2, \mathrm{~s}_{2}= \pm \sigma / 2 \\
& \text { for } n=3, \mathrm{~s}_{3}= \pm \sigma 2 / 7^{1 / 2} \\
& \text { for } n=4, \mathrm{~s}_{4}= \pm \sigma \\
& \text { for } n=5, \mathrm{~s}_{5}= \pm \sigma(8 / 5)^{1 / 2} \\
& \text { for } n=6, \mathrm{~s}_{6}= \pm \sigma(5 / 2)^{1 / 2} \\
& \text { for } n=7, \mathrm{~s}_{7}= \pm 2 \sigma \\
& \text { for } n=8, \mathrm{~s}_{8}= \pm \sigma 7^{1 / 2} \\
& \text { for } n=9, \mathrm{~s}_{9}= \pm 4 \sigma
\end{aligned}
$$

where $n$ represents the symmetry modulus or degree of freedom giving birth to each World Plane of the Omniverse.

\begin{tabular}{l|l|l|lllll}
\hline 0 & $1 / 2 \sigma$ & 1 & 1 & 1 & 1 \\
$1 / \sqrt{7} \sigma$ & $\frac{1}{8 / 5 \sigma}$ & $\sqrt{5 / 2} \sigma$ & $2 \sigma$ & $\sqrt{7} \sigma$ & $4 \sigma$ & $s$
\end{tabular}

Fig. 2 Primeval sequence overscale of degrees of freedom laid on the s-axis

These values are expressed in terms of $\sigma$, the standard deviation of the Omniverse (Fig.2). To the limit, one may tend to view each of these values of $s$ as a constant of motion in the traditional sense of a constant representing an invariant anchor. However, they are indeed best conceptualized and presented as the variants of motion, or the basis variant degrees of motion (since they typify variance). In this study of the origin and description of motion as solely resulting from behavior of space spreads, let's formally designate them the primitive variants of space momentum. They represent first-order descriptors of motion or precursors of motion. As such there is nothing beyond them, except for the order of dimensional modulus. Each of these variant primitives determines a manifold or eigenform, and a form of motion which concomitantly precurses a degree of motion, or an eigenkinesis. In other words, there exists a basis rate of motion

- in all circular motion and quantified by $s_{1}$.

- $\quad$ in all elliptic motion and quantified by $s_{2}$

- $\quad$ in all parabolic motion and quantified by $s_{3}$

- $\quad$ in all hyperbolic motion and quantified by $s_{4}$ and so on and so forth until the linear 9hyperbolic form of motion quantified by $s_{9}$.
So therefore every root form is associated with a root level of motion, a form being the contour or topological geometry tak-

\begin{tabular}{|c|c|c|c|}
\hline Distance Qty & $\mathrm{m} / \mathrm{s}$ (Scientific) & $\mathrm{m} / \mathrm{s}$ & Fraction of $c$ \\
\hline \multirow[t]{3}{*}{$1 \mathrm{~nm}$} & 1.00000000E-09 & 0.000000001 & $3.336 \mathrm{E}-18$ \\
\hline & $1.00000000 \mathrm{E}-08$ & 0.00000001 & $3.336 \mathrm{E}-17$ \\
\hline & $1.00000000 \mathrm{E}-07$ & 0.0000001 & $3.336 \mathrm{E}-16$ \\
\hline \multirow[t]{3}{*}{ 1micron } & $1.00000000 \mathrm{E}-06$ & 0.000001 & $3.336 \mathrm{E}-15$ \\
\hline & $1.00000000 \mathrm{E}-05$ & 0.00001 & $3.336 \mathrm{E}-14$ \\
\hline & $1.00000000 \mathrm{E}-04$ & 0.0001 & $3.336 \mathrm{E}-13$ \\
\hline $1 \mathrm{~mm}$ & $1.00000000 \mathrm{E}-03$ & 0.001 & 3.336E-12 \\
\hline $\mathrm{cm}$ & $1.00000000 \mathrm{E}-02$ & 0.01 & 3.336E-11 \\
\hline $1 \mathrm{dm}$ & $1.00000000 \mathrm{E}-01$ & 0.1 & $3.336 \mathrm{E}-10$ \\
\hline $1 \mathrm{~m}$ & $1.00000000 E+00$ & 1 & $3.336 \mathrm{E}-09$ \\
\hline $3 m$ & $3.00000000 E+00$ & 3 & $1.001 \mathrm{E}-08$ \\
\hline $3 \mathrm{dkm}$ & $3.00000000 \mathrm{E}-01$ & 30 & $1.001 \mathrm{E}-07$ \\
\hline \multirow[t]{3}{*}{$3 \mathrm{hm}$} & $3.00000000 \mathrm{E}+02$ & 300 & $1.001 \mathrm{E}-06$ \\
\hline & $3.00000000 E+03$ & 3000 & $1.001 \mathrm{E}-05$ \\
\hline & $3.00000000 E+04$ & 30,000 & 0.0001001 \\
\hline $300 \mathrm{~km}$ & $3.00000000 E+05$ & 300,000 & 0.0010007 \\
\hline $3000 \mathrm{~km}$ & $3.00000000 E+06$ & $3,000,000$ & 0.0100069 \\
\hline \multirow[t]{2}{*}{$30,000 \mathrm{~km}$} & $3.00000000 E+07$ & $30,000,000$ & 0.1000692 \\
\hline & $7.50000000 \mathrm{E}+07$ & $75,000,000$ & 0.2501731 \\
\hline \multirow[t]{5}{*}{$150,000 \mathrm{~km}$} & $1.50000000 \mathrm{E}+08$ & $150,000,000$ & 0.5003461 \\
\hline & $2.25000000 \mathrm{E}+08$ & $225,000,000$ & 0.7505192 \\
\hline & $2.90000000 \mathrm{E}+08$ & $290,000,000$ & 0.9006231 \\
\hline & $2.99000000 \mathrm{E}+08$ & $299,000,000$ & 0.9973566 \\
\hline & $2.99700000 \mathrm{E}+08$ & $299,700,000$ & 0.9996916 \\
\hline $299,792 \mathrm{~km}$ & $2.99792458 \mathrm{E}+08$ & $299,792,458$ & 1 \\
\hline
\end{tabular}
en by the quanta within the spread of space bearing the particular variant primitive or eigenkinetic primitive. In the Omniverse, we know that $s_{3}$ is the eigenkinetic primitive that precurses the degree of motion set at $0.299796065 \sigma^{-1}$, which in turn precurses the degree of motion materially realized at $c$ in our 3-D World Plane (the physical quantity we have labeled $299,792,458 \mathrm{~m} / \mathrm{s}$ ).

Table 1. A list of SI speed quantities in different formats expressed as a function of $c$, toward a nomenclature standard

We show in Table 1 a list of SI speed quantities expressed as a function of $c$. More than a limit, $c$ is the beginning of observable motion in our World Plane, because it is therein the first of all quantities of motion physically realized beyond the primitive. One way to understand $c$ is in terms of an order of space momentum, as a surrogation of the Newtonian concept of momentum. In this case it would be the product of a 
stress quantity times a motional quantity. We do not wittingly indulge in that representation at a formal level however, only invoking it as a helper to visualization in relating to older more familiar concepts. Further on in this study, we propose a more consistent interpretative approach to the quantification of $c$. Speed $c$ emerges as the most natural scale to measure motion in the 3-D World Plane, if only we understand that all observable objects's motion, no matter their modalities (angular or linear) and quantities, physically derive from it. From that perspective, it does not matter how complicated or simple the expression of all motions over the $c$ scale may turn out to be nomenclature-wise. The great advantage ensuing from adopting a measurement scale anchored on $c$ is that it affords us to be in tune or in synchrony with nature when it comes to kinetic instrumentation or transport technology, aside from absolute universality.

\section{Covariance vs. Causation}

The order of Quanto-Geometric covariance between scalar and space is not given in time, which is a variable the Theory irrevocably disavows and expressly bans. This account begs the question of the role of motion in covariance. In other words, does motion mediate covariance?

We must acknowledge that the first cause of covariance is conservation. Quanto-Geometric Theory postulates the exclusive duplet existence of a scalar tenet and a space spread tenet as the foundation of all physical objects' ontologies. Coexistence between the scalar and the intangible void represents the basis topological state of the Universe. The forced coexistence between the scalar and the vacuum spread gives rise to the first conservation constraint which sustains the foremost order of ontological covariance in the universe. Consequently the only fate of the two basis ontological tenets is to correlate with each other.

The scalar automatically inhabits the space spread so to speak. Therefore correlation is automatic and ineluctable between the two basis ontological tenets. While the correlation, in order to become covariance or causal interaction, implicates action, it does not implicate motion in the form of translation. Motion is already part of the ontology of space. One must expressly guard against visualizing motion as the end result of the interaction. However the motion of the scalar is indeed an expression of the interaction or the influence exerted on the scalar by space, while the tensorial stress experienced by the space spread represents the effect of the influence of the scalar over the space spread. The interaction or covariant action is not realized in time, which is here the big absent. The mutual interaction is exerted concomitantly. It has no beginning and no ending point, it is perennial and permanent. That is also the reason why there is in existence no pure scalar nor is there any existent pure space. All space is tensorial or under some level of stress and all scalar is spread in density state or exists in volumetric state.

Hence, the first cause is the coexistence constraint or conservation. Covariance or mutual prospection is the second level of causation. And the third cause of association or permanent association is the grip between the two tenets which establishes a degree or gradient of correlation.

Finally let's make sure to stress that if we were to insist in injecting time somehow in this program, because a paramount Quanto-Geometric axiom is orthogonality $(Q \times S=1)$

[1] [2] between the two ontological tenets (they are inverse of one another), their association with time would have to reflect their ontological orthogonal attribute [10]. Time for one tenet $(+t)$ would have to be the conjugate of time for the other $(-t)$, which would cancel time altogether out of the interaction. Therefore as quanto-geometrically framed, there is no place at all for time in this program. In consequence, motion cannot be expressed in this framework in terms of time in any manner whatsoever.

\section{Scales of Motion}

The program that we have described above represents the first-order conditions of motion in the 3-D physical world and directly precurses motion as it exists throughout the Omniverse. The correlation between universal space in every World Plane of the Omniverse and its total scalar mass represents the phase order from which motion is orchestrated as a direct native from the dimensional set defining every specific World Plane. Therefore each World Plane as a whole owns its own precursor of motion $c_{i}$ produced by the invariant quantogeometric degree of correlation between its mass and space proper, which conforms the symmetry basis for the transformations the specific World Plane will develop as a matter of its phenomenological circumscription.

Consequently, the first dynamic system in a World Plane is the entire World Plane itself and that horizon is the origin of the metric and all metrics. Everything else in a World Plane is a product of degeneracy of the specific determinations emanated from this global order and a local emulation of the global norms, including the norms of motion. 


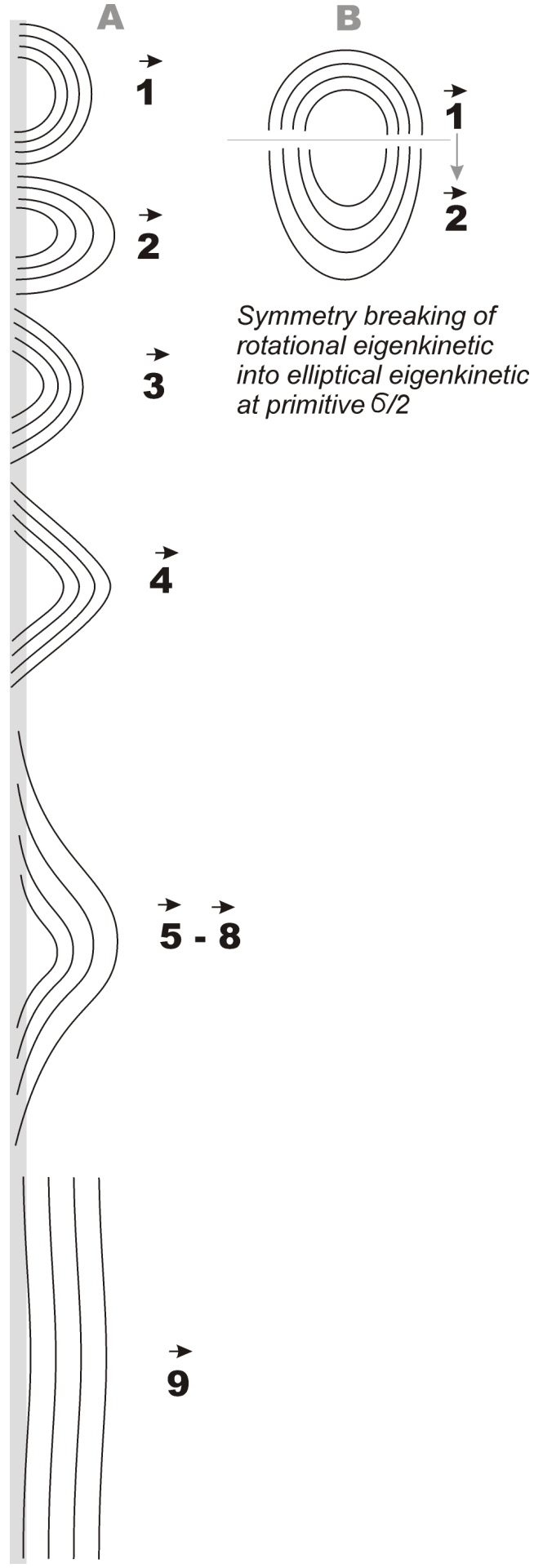

Fig. 1b. Representation of eigenkinetics within the tapestry of the vacuum (A). While one may liken them to geodesics, they in fact materialize the irreducibles of motion but not paths of motion in time. When a symmetry breaks, not only a new eigenform emerges but correlatively a new form of motion as well, as illustrated in (B). One shall keep in mind the very important fact that the represented eigenkinetics are intangible in nature.
Accordingly every eigenform [1] [2] relates to an eigenkinetic degree originated from its kinetic primitive (Fig. 1b). Each of these correlations is pre-eminently featured by a World Plane of the Omniverse, but the specific dynamics of a World Plane occurring below the dimensional background are developed according to an emulation of the whole primitive panoply in conjunction with the main Operator Norm in action for that Plane.

The next order of dynamic systems right below the global World Plane horizon is the order of Shells [1]. We are not going to delve here into the analysis of the dynamics of Shells of Matter, in particular that of the 3-D World Plane of most interest. We are only invoking it here in order to represent the order of dynamic systems as they truly occur in matter, quite differently from what we have normally conceived as a dynamic system thus far in all-time physics. Again each Shell is based on its own fundamental invariant sustaining the transformations circumscribing its phenomenological framework. It is only at that level that gravitation, a matter of utmost interest currently in the field, arises with the constant of Gravitation $\mathrm{G}$ as the fundamental invariant native to a particular Shell of the 3-D World Plane. These regimes of motion are modeled by quanto-geometric covariance mathematically represented in several functional differential orders essentially.

What we are going to do next is to describe how the primitives of motion reflect on well known dynamic scenarios in an effort to firmly falsify the above postulates.

\section{What is a Dynamic System?}

What we generally call a dynamic system is a scenario with at least two actors of scalar nature in motion of some kind with respect to one another. For instance, an atomic nucleus with a bound electron, or a star with one or several planets in orbit all ordinarily feature a dynamic system. A cat chasing a mouse we do not generally consider a dynamic system since the norms of motion thus far known in physics do not allow the interpretation of such systems involving the living. That scenario we would rather call an event. However, if the Quanto-Geometric Function says anything as a stochastic function, it is that it is well at the root of the expression of life and its dynamics as well.

It is important to understand at this point that each and every one of the 9 Quanto-Geometric covariant phases that put in correlation a scalar density entity and a space spread entity represents indeed a dynamic system. They each represent the most generically conceivable dynamic systems. They take the counter position to motion being the result of interaction between two mass-ive bodies as conventionally viewed and in- 
stead postulate motion to be a property of the space spread which engages the scalar, and in so doing creates a particular form of motion equally reflected in the morphology of the scalar object as a specific geometric eigenform. We have already explained the conservation constraints and the causal regime sustaining this dynamical order. The pictorial representation in (Fig. 3) serves to fix the idea, albeit not the most accomplished illustrative representation for the concept. It is based on the Ying Yang symbol which most of us under all latitudes have become familiar with in one degree or another.

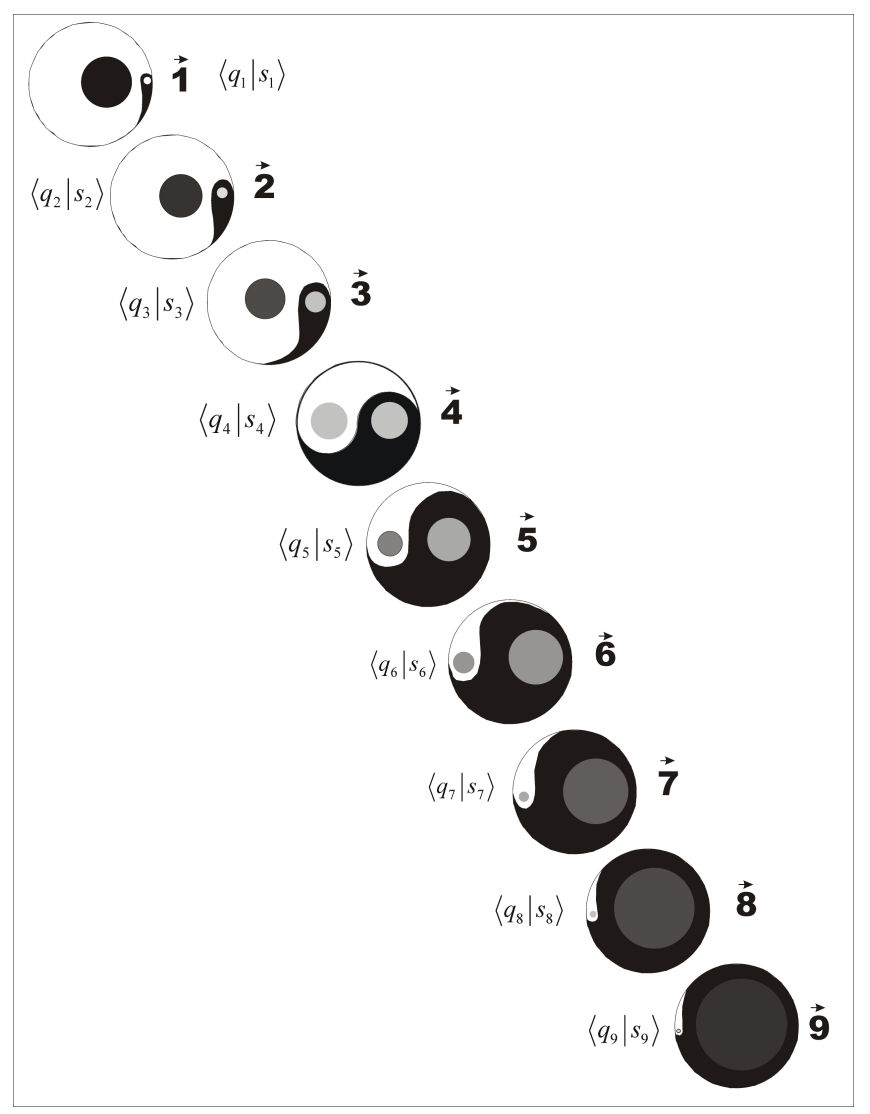

Fig. 3 A depiction of the primordial Quanto-Geometric ontological tenets and their covariant regime

So therefore a theory of gravitation as interaction between two bodies is very far and away from being the ultimate theory of motion in nature or one for generic dynamic systems. Einstein's Theories of Relativity have certainly taken the correct direction by establishing interaction between space and mass as the engine of gravitation. However, he had not properly viewed the intrinsic properties of space, in particular its inherent motional elements in their many different orders, quite aside from the successful characterization of the tensorial nature of space; nor has the Theory understood that the correlational trend between matter and space does not only exist in the cosmological realm but at all scales in the 3-D realm of matter [9].
The illustration in Fig.3, shows three different orders of representation. One is the scalar density order represented in the white spread in the diagram, the other is the space spread represented in the black spread in the diagram. Third order of representation is the correlational or covariant influence within the primary duet portrayed by the two inner gray-tone circles inside each spread. From the monolithic object to the transcendental object:

- Space spread evolves by augmentation within the object's ontology.

- The quantum scalar evolves by diminishing its presence within the object's ontology.

- The correlation evolves in a way that sees the scalar to be dominantly influential at one end, gradually loses influential weight while the space partner acquires more influential weight or becoming predominant. This is expresses in the grey tone and size of the inner balls.

Why do we say object and not a dynamic system? Because an object of any kind is intrinsically a stationary dynamic system. This framework replaces the expression of the First Newton Law with the parallel notion of quanto-geometric momentum $Q_{G}$, which stands for the product of the two basis tenet:

$$
Q_{G}=Q \times S
$$

where $Q$ stands for scalar density and $S$ stands for the motional artifact or the spatial wavefunction in their most irreducible state.

The least amount of motion is given in the monolithic circular eigenform as gyric motion. While the largest amount of motion is given in the transcendental eigenform as quasi rectilinear motion. It does not matter how fast a perfectly circular motion is realized, it has less motion than the most perfectly realized linear motion, simply because the expansive translational component of motion is at a minimum in circular motion. To make sense of that motional hierarchy, one may look at the eccentricity $\boldsymbol{e}$ of the eigenform related to each type of motion or its eigenkinetic.

- For the circle eigenform, $e=0$

- For the ellipse eigenform, $0<e<1$

- For the parabola eigenform $e=1$

- For the hyperbola eigenform $e>1$ 
- For the 5- to 8-hyperbolic eigenforms $\quad e>>1$

- For the rectilinear 9-hyperbolic eigenform $e \rightarrow \infty$

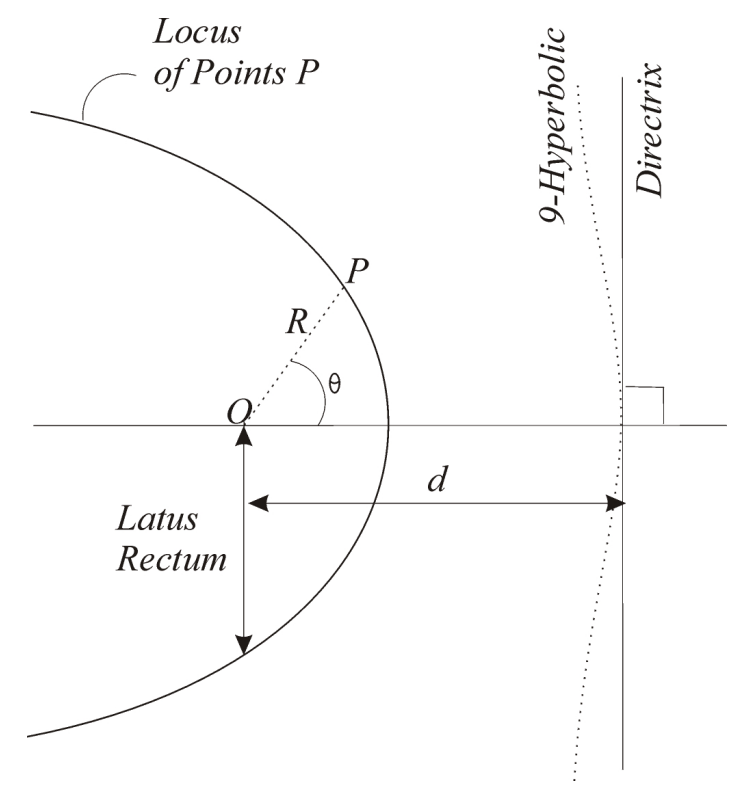

Fig. 4 Definition of a conic function

We assert that as the eccentricity increases so does the eigenkinetic value of the motion. The mother of all motion is the rectilinear eigenkinetic because it potentially gives birth to all other eigenkinetics. One can easily concur with this appreciation by comparing circular motion to linear motion. While there is indeed a linear component to the circular eigenkinetic, essentially given by the run over the perimeter of the circle, contrastingly there is no circular component to the most perfectly rectilinear eigenkinetic.

As a reminder, eccentricity is the constant defining the locus of points $P$ relative to a line called the directrix and a fixed point $O$, per illustration in Fig. 4 and the following equation:

$$
e=\frac{R}{d-R \cos \theta}, \text { where } e \text { is the eccentricity. }
$$

Keep in mind that while this characterization helps understand the primordial hierarchy of motion, it is not perfect in that it does not represent quite correctly the hyperbolic order of motion which lies between the hyperbola eigenkinetic and the rectilinear eigenkinetic [17]. It is only successful in helping characterize the transcendental hyperbolic eigenform or eigenkinetic, because the latter, by essentially having a life near the asymptote, can be practically assimilated to a rectilinear element, if $\sigma$ takes a sufficiently large value.
In this study of the different orders of motion, two remarks are important to put in highlight:

1. Because in Quanto-Geometric Theory, $s=0$ is undefined, absolute spin is not possible. There is always some level of precession in rotational motion. In that sense a circle is already a precessional figure.

2. Some clever minds have found a scale of $\pi$ where an object can make several full rotations before returning to its original starting position (spiral torus figure). That is simply an expression of $\pi$ in 3-space, specifically spin motion over a cylindrical surface, and can never be true in 2-space where all primitives of motion originate.

\section{Spin within the Hierarchy of Motion}

While spin is probably hitherto overrated in quantum physics, there is apparently a certain level of misunderstanding associated with spin motion as well. As previously mentioned, absolute spin is not possible, but if it existed it would the first gradient of motion. Absolute spin is rotation without translation or precession. One cannot imagine or represent motion of any kind in non-precessional spin. You can only represent spinning motion from the portrayal of its edges, the precessional perimeter. Once this image disappears one can no longer represent the amount of motion in spin, simply because the idealized center of spin experiences no motion whatsoever. Hence counter-intuitively, the state of zero-motion or gradient 0 of motion is indeed the state of absolute spin. Its physical realization (of provenance $s=0$ ) is indeterminate or forbidden in Quanto-Geometric physical law, making it thus an impossible.

We characterize idealized zero motion as rest. In practice, we know full well that absolute zero motion or absolute rest is not possible, not because of the relativistic nature of observational frames, but because there always exists a framework of transformation to overtake any apparent object at rest. Consequently two objects can only be at apparent total rest with respect to one another because they are moving identically in every factor or component of motion. This situation materializes the closest image possible to absolute spin. It is because absolute spin represents gradient 0 of motion that the next step in the hierarchy of motion (indeed the least countable one) is precessional rotation incarnated in the figure of a circle and rated by the $\pi$ constant. 


\section{Linear and Harmonic Motion in the Quanto- Geometric Regime}

Traditionally, one studies linear and curvilinear motion in a distinct chapter than harmonic motion [15] [16]. However Louis Victor De Broglie's quantum relativity has eloquently shown that all motion, at least all objects in linear motion, are concomitantly subject to a harmonic motion as well [5]. In Quanto-Geometry the eigenform that pairs with an eigenkinetic is a direct expression of the wavefunction because the $e i$ genform is indeed a physical waveform. Consequently, and in accordance with Quanto-Geometric 2-space metric, every primitive of motion is a descriptor for both a degree of translational motion and a wavelength defining a wavefunction.

We have to abandon the idea that all wavefunctions, which we indiscriminately model on the eternal sinusoid, are the same. All wave functions are not the same because the symmetry implicated in their harmonicity (cyclic repetition) is based on different eigenforms, which are again true material waveforms. The only eigenform or eigenkinetic that can model all other eigenforms or eigenkinetics is that one belonging to the Quanto-Geometric transcendental (9-hyperpolic) layer. One can see that as $\sigma$ becomes very large the QuantoGeometric Function graphically approximates a line and as all parameters rescind, the function migrates into a circle.

The generic Quanto-Geometric Operator, which stands for an alternative representation of the Grand Eigenfunction portrays the best mathematical expression for modeling dynamic systems. It outwardly displays in the habits of real quantities, estranged from all imaginaries or abstracts, the two components responsible not only for ontology but for phenomenological interaction, that is to say the wavefunction and scalar density tenets. The exponential component in the expression stands for the wavefunction while the $Q$ term stands for the scalar:

$$
\|\psi\|_{a_{i} b_{i}}=\left[\frac{-Q^{2}}{2 q}\right] \cdot\left[\frac{2}{\left.a_{i} e^{\left(\frac{2 a_{i}+b_{i}^{2}}{4}\right)}+b_{i} e^{\left(\frac{2 a_{i}+b_{i}^{2}}{4}\right)}+c e^{\left(\frac{2 a_{i}+b_{i}^{2}}{4}\right)}\right]}\right]
$$

Every sub-index $i$ in $a_{i} b_{i}$ represents a $q-s$ set, making up either an object or the tenets of a dynamic system. The wavefunction component is an expression of what we call Potential Energy, whereas the scalar term represents what we normally call Kinetic Energy. It is clear that Quanto-Geometric Theory radically concurs with the perspective of Hamiltonian physics which views Energy as the primary state of physical objects, and not the Momentum/Force perspective offered in Newto- nian physics. An important rectification is required however, which mandates the scalar term to stand for the Potential Energy proper since it is innately static, while the exponential term ought to stand for Kinetic Energy, since it represents the spatial function, the innately kinetically active component.

\section{Deployment of the Panoply of Motion Primi- tives}

As we have previously explained, the super-imposed metric on the scale of 2-space marks the origin of the gradients of motion. The elements of this metric constitute the primordial gradients for motion both in their qualitative and quantitative characterizations. The quality means the morphology taken by motion along the scale of rectilinear at one end to circular at the other end. Worth reiterating that this includes the characterization of waveforms, deriving into the ordinary concept of wavefunctions. The primitive quantities range from limiting values in the neighborhood of $0^{+}$to $1 / 2 \sigma$ to $1 \sigma$ all the way up to $4 \sigma$ (Fig. 2).

Let us examine a typical dynamic scenario in the manner experienced by an electron in a potential well created in the laboratory in the form of an evacuated chamber (i.e. our old cathode ray TV sets), or a chamber filled with mercury gas, per the original Frank-Hertz experiment (Fig.5). In our view, the well and the electron form a dynamic system of secondorder quanto-geometric nature.

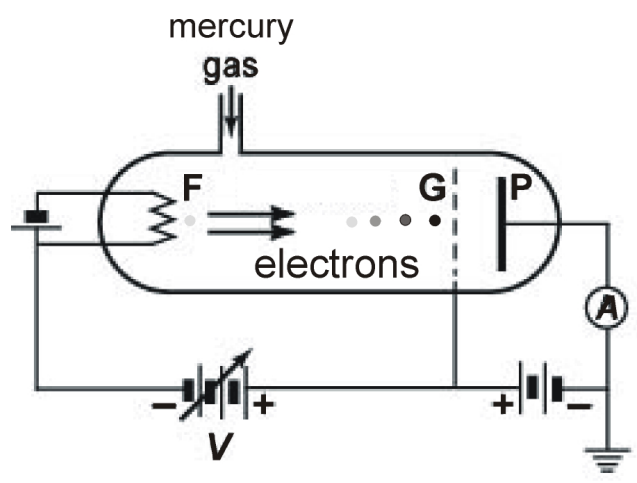

Fig.5 Franck-Hertz experiment of travel electrons in a mercury-gas filled chamber

The experimental setup has a cathode $\mathrm{F}$ and an anode $\mathrm{G}$ inside of a gas filled crystal tube (or an evacuated tube) create an electric field which steers the electrons created at the cathode to the anode. Classically, the potential well should be able to cause the electrons to move at any speed value and in a linearly proportional manner to the energy quantity communicated 
to the electron by the electric potential. However, the dynamic outcome is much different than the classical expectation.

What we see per Fig.6 is a quasi typical quanto-geometric distribution $^{2}$ that correlates Kinetic Energy to particle speed. We interpret that the potential well supplying kinetic energy to the particle in the form of electron-Volts $(\mathrm{eV})$ over-powers or provisions a boost to the innate wavefunction of the particle and thereby causes an imbalance in the inner covariant scalarspace phase inherent to the particle. The particle reacts to this imbalance with a covariant shift re-balance, which is naturally reflected in the space momentum of the particle across the well, in accordance with the distributive disposition of the Quanto-Geometric Grand Eigenfunction. Why the limit in the accessible degrees of space momenta is $c$ is a different matter, which we already discussed. The limit per se is imposed by the distribution. Here the potential energy from the potential well, which the particle experiences kinetically, plays the role of the primitives of motion. It can take any value in the absolute but there is an effectual metric imposed on it which is the one that is going to be permeable to the particles and reflected in the space momenta they develop. To put it simply, the particles redistribute their ontological quantum-space composition per the Grand Eigenfunction's 9-layer norm.

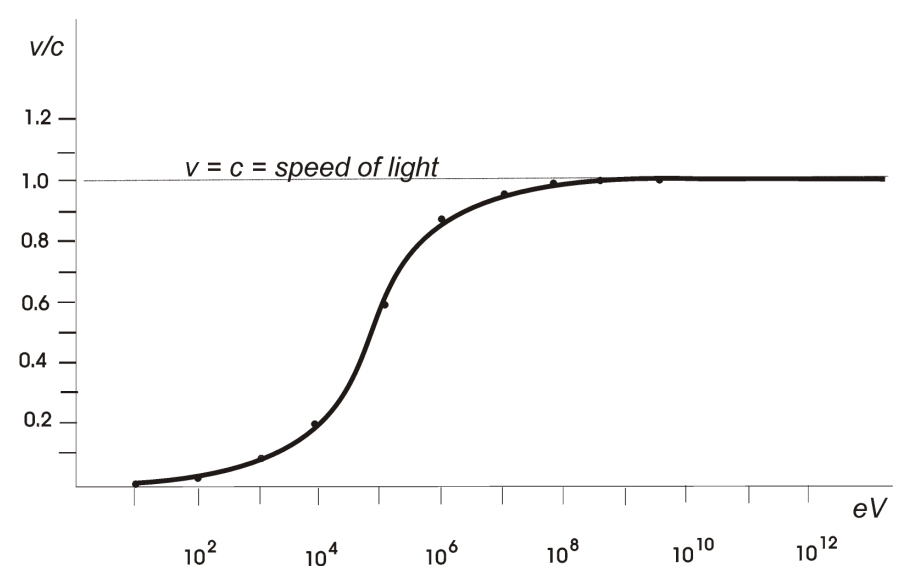

Fig. 6 Distribution of particle speeds vs their acquired kinetic energy

Finally, it is worth noting that the reason why one sees here a somewhat skewed quanto-geomeric distribution is because of a somewhat irregular scalar-space covariant shift within the travel particles ultimately due to the way kinetic energy is communicated to the latter. We see a similarly

\footnotetext{
2 This graph corresponds to the preliminaries of the Franck-Hertz experiment whereby the applied voltages fall outside of the range that would famously cause the first-time observed quantization of the energy levels of the electron per its inelastic interaction with the mercury gas molecules. These voltages are significantly higher than those causing quantization behavior.
}

skewed pattern when optical energy, instead of electric potential energy, is communicated to the particles, as displayed in the photoelectric effect experiment (Fig.7).

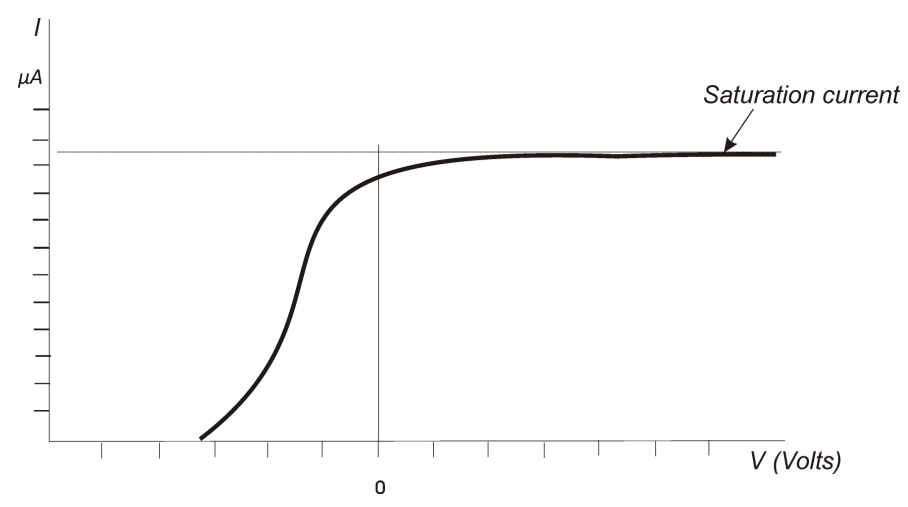

Fig. 7 Photoelectric experiment where incident monochromatic rays on a metal plate induce an electron flow or current.

In this experiment, the electric potential still develops the primitives of motion versus the current $A$ or flow rate of electron particles ejected from the cathode. The quanto-geometric distribution of the curve is similarly skewed akin to the case previously discussed, as a particular effect of the manner the energy is communicated to the particle, here thru monochromatic light $(h v)$. The skew in both cases may be studied in terms of an effect on the $\sigma$ parameter of the distribution.

Also note that travel electrons in interstellar space will presumably demonstrate the same type of distribution reported in Fig. 4, as do other particle objects such as protons. This pattern is universal for all solid objects made to absorb energy in a way that would cause a change in their momenta.

\section{Transfiguration of Speed in Space Momen- tum}

All speed is rate, but the nature of this rate is essentially and uncharacteristically a density function. Not just in the mathematical sense, but very physically so. The de-generate deployment of space from $c$ indeed creates the observable speed of objects as the first level of motion, whose universal etalon in the 3-D World Plane is $v / c$, and not a time-based rate. Because all observable motion is a normalized density function, the rate $v / c$ is maximally set at 1 and 1 is an unrealizable, in accordance with one of the foremost Quanto-Geometric prescriptions. In other words, $v=c$ is unachievable for solid or fermionic objects. If that fact was already given by other evidence, then we must further understand that the reason why that is so is because motion is a Quanto-Geometrically distributed deployment. 
Analysis of objects' statistical topological states has been realized in [3] and will not be reproduced here. In strong support of the above, we will here report, however, the graphical Fermi-Dirac distribution, which is a faithful copy of the distribution obtained in the preliminaries of the Franck-Hertz experiment for the travel electron.

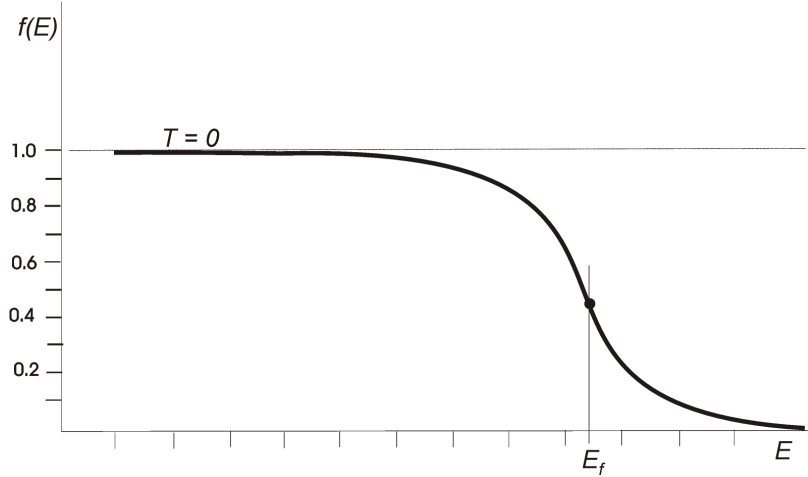

Fig. 8 Probability distribution of occupation of energy states by a topology of free electrons

Even in topologies of objects, which altogether conform a sub-Shell, motion is quanto-geometrically distributed with thermodynamic temperature as the standard deviation parameter. In this scenario, motion is the motion innate to physical states. The function $f(E)$ in Fig. 8 represents the probability of occupation of energy states by individual electrons. The energy levels are the primitives of motion. It is not at all surprising to see here that most particles tend to occupy the lowest level of the energy (curve flipped from right to left as compared to Fig.6), because it is here about a topology or a stateful act, not an expansive act of translational motion. However, the normal nature of the $f(E)$ function (with 1 as the higher limit), informs us by analogy of the density nature of the $v / c$ space momentum function for the same elements in travel instead. The empirical formula for this function, below reported, is important when it comes to numeric analysis, but not so in the quest for an explanation to the cause of the behavior it describes:

$$
f(E)=\frac{1}{e^{\left(E-E_{f}\right) k T}+1}
$$

By the same token, this parallel allows us to visualize the physics that we invoked when we established a line of equivalence between stateful objects and dynamic systems.

\section{What Creates Lorentz Invariance and the II- Iusion of Time?}

An important figure in relativistic physics is what has been called the $\beta$ ratio, the ratio of any attainable speed versus the referential speed of light:

$$
\beta=\frac{v}{c}
$$

In the following, we are going to clarify the meaning of this ratio as a function whilst making use of it in order to uncover the origin of continuum physics, where time is king, along with the foundation of Lorentz invariance.

\section{Basis of Continuum Physics}

Because of our inability to visualize the origin of motion in terms of the panoply of primitives of motion, we have been arguably misled by a first-order derivative of the Function that relates those primitives to the development of actual velocities or speed quantities. A graphical view of the derivative of the Function is shown below in Fig 9.

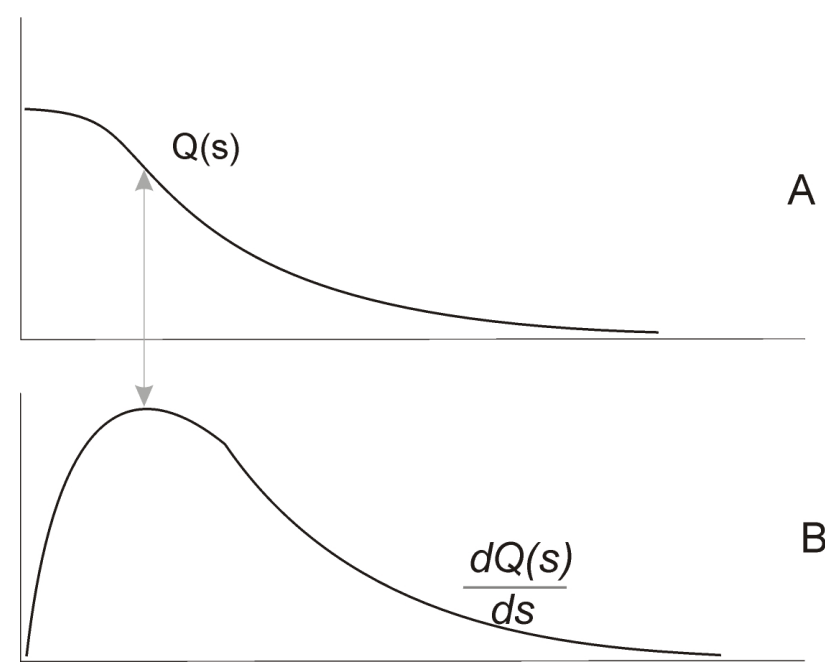

Fig. 9 Derivative of the Grand Eigenfunction

If we let the derivative represent classical momentum $(\mathrm{m}$ $d x / d t$ ), one can clearly see that in the region of momenta neighboring 0 , for one value of the derivative there are, instead of two singular values of the primitives, two corresponding quasi sets of values of the primitives: the values of $s$ approaching $0^{+}$, which are continuously varying fractional quantities, and the values of $s$ beyond $4 \sigma$, which behave linearly in the neighborhood of the $s$-axis asymptote and constitute equally continuously varying quantities. This effect is even more pronounced in the skewed distributions that we have reviewed above as we examine their functional derivatives. It is indeed thru this window that we have historically been looking at motion in nature, which has caused us to create and visualize time as this continual varying artifact playing the role of background substrate to motion in general. That also stands for the reason why we have argued elsewhere [18] that time works for us because we are slow creatures in a slow world of ours. As soon as we leave these regions of continuity, classical physics 
and common sense start breaking down, unable to properly account for common real-world motion because they no longer obey continuum (time) span.

\section{Basis of Lorentz invariance}

We may gain further insights in large scale physics if we assimilate the density function $\Delta r / \Delta s$ to the vertical axis of the derivative graph in Fig. 9. Because we are parts integral to the Universe as internal elements, and because we are a product of derivates of functions of motion, we cannot ascertain an extrinsic view of motion in the Universe as if a view of the same from outside, but only an intrinsic view of motion from the boundary of $v / c \rightarrow 0$ to which our living parts confine us, we thus cannot naturally become aware of the intermediate levels of the scale of the primitives and their corresponding space momenta.

In fact, what nature has done is to utilize our inability to perceive the upper region of the $\beta$ ratio in order to fashion our very important sense of sight. Effectively, the sense of sight for most living entities in the biosphere is based on generalized Lorentz invariance in all directions. At whatever speed an object is moving with respect to us and whatever speed we are moving with respect to the object, we will always see or perceive the object with complete definition, with no blur or aberration, that is, in a demonstration that perception is based on the invariance of the speed of light traveling from objects under observation to us. The same is altogether true for other simultaneous observers of the same object with a different kinetic experience in their own observational frames. Whereas our sense of motion naturally remains under the Galilean regime because nature has recognized that we need correct estimation of motion of common objects in our environment relative to us in order to conveniently keep ourselves safe or motionally efficient within the environment [18]. When we see an object in motion, there is two different levels of awareness being exercised relative to the moving object: there is the visual perception of the object and there is an innate perception or sense of the kinetic progress or displacement of the object.

Those facts, which are seldom understood and invoked in justification of the generalization of Lorentz invariance, constitute a square rebuke to neo-classical arguments denying that postulate, often coming from dissident physicists who do not realize that their argument is a bargain against their own sense of sight, without which they would be most likely unable to develop and hold the very argument!

The transformation technique that we use as an analytic method to make sense of Lorentz invariance is vastly imperfect and overrated, because, among other shortcomings, it does not allow us to analyze transversal motion of light from several simultaneous observers' vantage points. We can only visualize, physically and mathematically, a transformation in one single direction originating from the source frame. How to do it in a multitude of directions at a time, since any light travel event may be viewed simultaneously by a multitude of observers with same or different kinetic experience proper, remains out of reach, because in my sense it is ultimately utterly difficult to explain what bloody property or properties of the basis underlying spacetime allow a simultaneous sum of timelines with different time arrows and flowing at different rates and dilating at different increments to be totally transparent to one another. So therefore the analytic method of transformations of reference frames, otherwise attempted historically in more other ways than one can count, are ostensibly unable to thoroughly explain Lorentz invariance in all directions, leaving it to be postulated as a Special Relativity axiom.

The traditional translational transformation does little more than cover a game of running an inflation of time in correlative proportion with the Lorentz factor so that the speed of the perceived quantum of light always remains the same. An explanation of Lorentz invariance thru this overhead construction is, to say the least, somewhat artificial and very much inadequate. Matter-of-factly, to understand what makes the principle of Lorentz invariance valid in its generalization in all directions, and what makes the speed of light an independent invariant from all relative environmental scalar movement (a non-additive motion), one ought to invoke the kinetic properties of the underlying spatial tapestry and comprehend what makes the $v / c$ ratio a density function. This ratio is an expression of the measure of displacement of the scalar with respect to the displacement realized within the spatial tapestry, since it is the internal kinematic of the spatial vacuum that induces the motion of the scalar. The perennial defoliation or propagation of space from within its own tapestry and in all directions simultaneously, of which light is only a witness, is the preeminent cause of all motion and thereby casts all motion as a ratio of displacements. This ratio is indeed the amount of displacement experienced by the scalar object (or bundle) versus the amount of natural displacements implemented by space in the World Plane, our $-\Delta r / \Delta s$ figure. The scalar obviously can never experience larger displacements than the referential spatial displacements. Therefore the density expressed by this ratio is normalized at 1 . In addition, the displacement experienced by the scalar is a direct measure of the mass-ive gradient of the scalar. That stands for the reason why we see from the treatment thru the Grand Eigenfunction that what we know as the speed of light is ultimately rendered as the value of the (trinitary) scalar in the 3-D World Plane: 


$$
q_{3}=299,792,458
$$

An equivalent sign $\equiv$ would have been a better translation to the underlying physics, but this slight mathematical mischaracterization remains innocuous and bears no further consequence.

Absent a representation of the intimate kinematic of space in these terms, the $\beta$ ratio cannot acquire its full functional meaning beyond its appearance as a flat dimensionless ratio. What's more, one need not represent geodesical lines in the tapestry of space to describe motion, once it is understood to be the displacement ratio we described. The relativistic geodesics constitute a support for describing motion in time with the 4-vector $\vec{v}$ :

$$
\vec{v}=\frac{d x}{d t} \vec{u}_{x}+\frac{d y}{d t} \vec{u}_{y}+\frac{d z}{d t} \vec{u}_{z} .
$$

In our perspective, the geodesics are unnecessary. While one may liken the eignekinetics to geodesics (Fig. 1b) to some extent, the former in fact materialize the irreducibles of motion but not paths of motion in time. All trajectories of motion are determined by a unit-form of motion and a precursory quantitative schedule for that transport in the form of spatial variance or multi-variation.

\section{Eigenkinetic Velocity Vector}

In contrast to geodesics, we represent the eigenkinetic velocity vector to be a multivariate displacement in true coordinate space, as function of one observable spatial variable $s$ or two observable spatial variables $t$ and $m$, directly from our metric nomenclature [1] [2]. The expressions of the primitive velocity vector or eigenkinetic follows:

$$
\vec{v}_{i S}=-\left|\frac{d r}{d s}\right| \vec{u}_{s}, \text { in 2-space with } s \text { and } r \text { spatial coor- }
$$

dinates

$$
\vec{v}_{i M T}=\left[-\left|\frac{d r}{d m}\right| \vec{u}_{m}\right]+\left[-\left|\frac{d r}{d t}\right| \vec{u}_{t}\right] \text {, in 3-space with }
$$

$r, m$ and $t$ spatial coordinates, where $r$ is the scalar displacement coordinate and the $\vec{u}_{i}$ the unit-vectors along each axis of the coordinate system. The displacement rates are negative because the scalar moves in opposite direction to the inducing spatial displacement, in accordance with the prescription emanating from the first-order derivative of the Grand Eigenfunction, a negative quantity. Effectively, there is a direct isomorphic relationship between $d q / d s$ and $d r / d s$. In addition, the $i$ - values taken by the velocity vector pertain to the basis $s$-line of the metric, not to the real number domain.

The spatial velocity of the object is a tangent vector to the path, just as the eigenforms are representatives of curvilinear tangent bundles in the metric. On that account the eigenkinetic velocity vector parallels an eigenform. In other words, as primitives that spring from the $s$-line, the eigenkinetics walk hand in hand with the eigenforms as a specific eigenform, whether a 2-space or a 3-space manifold, composes the path of the motion in question.

It is of utmost importance to comprehend that the quantitative capabilities of the $s$-line metric, basis of the eigenkinetic primitives, are what confers to this descriptive framework at very first order intrinsic kinetic quantification, in discard of the traditional velocity measures in terms variations in time. Motion here is described over true coordinate space background and not an artificial spacetime construction.

\section{Effects of Motion on Mass and Mass Beha- vior}

Let us further examine the effects on mass inside of the scalar-space phase responsible for the momentum. One shall keep in mind that the phase shift implies an ontological displacement of the correlation, which means a covariant change in scalar density or mass of the particle and the correlated space spread of its makeup. This sweeping change in mass (or changing mass) is what the Theory of Relativity proposes for relativistic mass as the speed of the particle reaches the vicinity of $c$. As we know, the concept of relativistic mass is not without its caveats and controversies. In our sense, the reason for the controversy is simply because thus far in physics we have not understood mass to be defined as scalar density instead of a punctual monolithic thing lacking a primary or generic definition. In addition, for an object to have additional intrinsic mass with respect to an observer, as one interpretation has it, is plainly unsound philosophically. A. Einstein admitted that much about relativistic mass:

"It is not good to introduce the concept of the mass $\boldsymbol{M}$ of a moving body for which no clear definition can be given. It is better to introduce no other mass concept than the "rest mass" $m$. Instead of introducing $M$ it is better to mention the expression for the momentum and energy of a body in motion.

In other words, he unequivocally disavowed the expression of relativistic mass $M$ : 


$$
M=\frac{m}{\sqrt{1-\frac{v^{2}}{c^{2}}}}
$$

where $v$ is the registered speed of the object, $m$ its rest mass, and $c$ the speed of light. Most physicists acknowledge that the experimental results taken to seemingly justify the hypothesized concept of relativistic mass increase may well be subject to other forms of interpretation implying no mass increase at all.

Gravitational mass, inertial mass, relativistic mass, etc., are all conceptual models of behavior of mass which the real ontology of particles and objects knows nothing about. No object or particle has a mass that changes its nature according to the dynamic system where it is kinetically participating. Furthermore, one ought to wonder, if the mass, conventionally thought to be a monolith, is going to increase, where would the supplemental amount of mass be coming from? Not from thin air (or thin space!). Energy conversion from the principle of mass-energy equivalence is just another contentious proposition heavily litigated in recent past, which would further complicate the matter instead of providing clarifications. Relativists have thus settled for the safe interpretation suggested by A. Einstein. As a result, the nomenclature only ventures to state that as an object approaches the speed of light, the object's energy and momentum increase and without bound, while offering the following two supporting formulae:

$$
\begin{aligned}
& p=\frac{m_{0} v}{\sqrt{1-\frac{v^{2}}{c^{2}}}}, \text { (3) where } p \text { is the momentum } \\
& E^{2}=\left(m_{0} c^{2}\right)^{2}+(p c)^{2} \quad \text { (4), where } E \text { is total Ener- }
\end{aligned}
$$

gy, $p$ the momentum of the travel object and $m_{0} c^{2}$ the energy at rest, negligible relative to $p^{2} c^{2}$ if the object is moving at very high speed.

Note that expression (4) is derived from (2), as a result of multiplying the latter by $c^{2}$, despite the fact that (2) is expressly disavowed. To remain truthful and avoid the evident clever play of hide and seek with these concepts, one ought to acknowledge that if there must be an increase in Energy and momentum as the solid or fermionic object approaches the speed of light, there must be a loss in mass rather. What we recognize in Quanto-Geometric Theory is that although the value of the mass of an object is perennial in invariant conditions, transformations applied to the object, such as acceleration in potential wells may change the value of the mass. In particular, when the mass increases, the object has experienced an augmentation in its scalar density, when the value of mass decreases, the object has experienced a drop in its scalar density [3]. There is of course an allotted range for these changes, since beyond each of the primitive points of the super-metric, symmetry breaking occurs which transmutes the object into the next one up in the scale.

This view is strongly supported by the fact that all particles's mass-driven interactivity at speeds very near $c$ is highly diminished and subsequently at $c$ they transmute into vector bosons. Their mere natural conversion into vector bosons in spontaneous disintegration processes equally advocate for this view to a certain extent. The likely cause of this behavior is that $c$ is not just an imposed or arbitrary speed limit but in reality an endogenous kinematic implementation of the vacuum, which then completely subsumes the travel particle by shifting its wavefunction [3] and potentially volatilizing it altogether into void itself, thus leaving the particle with no mass-like capabilities. This view is fully consistent with the structure and morphology of particles proposed in Quantum Relativity, according to which a photon is a much larger entity than any fermion, and obviously less dense from a scalar point of view. When a fermion like the electron explodes into a photon i.e., as it happens in the reaction particle-anti-particle pair (electron-positron), it is not behaving like a scalar monolith, in which case it would split into smaller monoliths, but as a density or distributed scalar, accessible to a state of larger volumetric ontology represented in photons, translating thereby a diminution in its original scalar density. When a neutral pion $\pi^{0}$, on the other hand, naturally disintegrates into two $\gamma$ photons instead of explosively splitting in scalar parcels, one has every reason to think that the instability of these particles is conveyed thru a distributive volumetric state (or decreased scalar density) in an active intrinsic kinetic process that transmutes the particle into photons. These are all mass-ive behavior consistent with scalar density ontology for mass.

Lastly we shall consider the behavior of bound electrons flying at relativistic speeds around atomic nuclei, whereby they create a stationary density cloud we denote orbital, additional support to our view of mass ontology and behavior. What's more, even the structure of the very electron in those conditions appears to be stretched out, if one ought to give credence to E. Schrodinger's view that the bound electron must be like a blob. To the same record, we must firmly add the fact that in those same conditions, the particle cannot demonstrate momentum or inertia, a mass-laden characteristic. 


\section{Time-free Experience of Motion in Quantum Entanglement}

Quantum Entanglement has historically caused quite a stir in modern physics due to its puzzling defiance of the notions of time and determinism. It is particularly opportune to address this conundrum in an exposé on the subject of timefree motion in dynamic systems and offer a new line of rational interpretation, quite apart from the atypical and contentious notion of retro-causation. From the EPR paradox, which was a thought experiment by A. Einstein, Podolski and Rosen, involving two photons receding from one another (obviously at the very speed of light), to the actual realization of the experiment in the 1970's to the now ample variety of possible experimental entanglement methods involving even atoms or molecules in lieu of photons, physicists have been driven to the same conclusions, ultimately leading to time as an entity emerging from quantum entanglement phenomenology.

\section{Definitions}

First a few definitions. An entangled system of a pair of quantum objects or particles is one in which the sum of internal components that make up the state of one object is insufficient for the complete description of the state of the same and is dependent on the state of its par. It is primarily not really about dynamical interaction but about state. That is why an entangled pair of quantum objects is said to in the singlet state. The state behavior of these objects, so to speak, appears to be that of a single unified object. Two bound electrons within an atomic orbital is the perfect example of a natural pair of entangled quantum objects.

The second condition for an entangled pair of quantum objects to be or become such is that they must be a conjugate of one another in some way: i.e. in the orbital habitat the electrons must be in the spin-up state for one and the spin-down state for the other. One may abusively sum up this condition by stating that the pair must have an opposite par quality to it.

\section{Wavefunction Collapse}

The gist to the matter here is that any circumstance that affects one element of the par such as a measurement of any sort (momentum, position, speed, etc.) automatically and instantaneously affects the other conjugate, and does so before any information could travel from the affected particle to its par (no signal can travel faster than $c$ the speed at which the photons are already receding from one another). The state of the entire entangled system collapses in these circumstances. Einstein et al contended that at their coherent inception both particles acquired unknown or hidden information about each other in relation to their local variables or shared the same determinants, which would help explain why they behave in a correlational manner without exchange of information of any sort. Bell's inequality theorem destroyed this view to the increasing satisfaction of the modern physics community by showing that on the experimental plane it does not pass the muster of the strict laws of probability because the measurement results remain unexpectedly always correlated.

As recently as 2013 entanglement has been obtained between photons that did not coexist in time in a demonstration of what has been called entanglement swapping. We must also point out that entanglement between quantum objects has been realized even when the measurements have been performed quicker than light could transit between the two entangled objects.

As much as the current conclusions driven from quantum entanglement experimentation found EPR position vis-a-vis statistical quantum mechanics erroneous, from the QuantoGeometric vantage we can fully support their contention that no aspect of three-dimensional physics can be outside of the scope of intellectual interpretation and that we must not accept no explanation for an explanation. Effectively, one will find no weirdness or mystery to the behavior of quantum mechanics' wavefunction as long as we are able to understand the higher order of Covariant Norms, unknown thus far to past and modern-day physics, in its 9 typified forms and its 3 quintessential forms, that is central to Quanto-Geometric Theory [1] [2]. It is only in the ignorance of the many properties innate to the void of space, beyond the geometric properties uncovered by Einstein's Theories of Relativity, that one will find mystery in the behavior of Quantum Mechanics wavefunction in general.

\section{Unraveling the Quantum Entangled State}

To be specific, an understanding of quantum entanglement starts with a physical visualization of the quantum wavefunction.

\section{Significance of the Boundless Spread of the Wavefunction}

Shall we say that it is time to make good on the quantum-mechanical concept of the boundary-free wavefunction. The quantum wavefunction is real and not an imaginary or abstract mathematical entity with a life in momentum space. The quantum wavefunction is indeed the spread of the void of space. For what we call a particle object, it may be visualized as a tensored regionalization of the void of universal space in the immediate surrounds of a point scalar, in the exact manner we have depicted it in the ying yang representation (Fig.3). It is not and cannot be a segregated expansion of background 
universal space, since it is integral to the latter. Its expression is the exponential term of the generic Quanto-Geometric Operator.

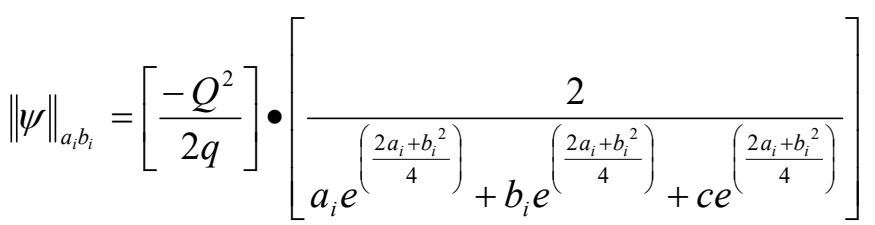

Therefore, from the standpoint of an object's wavefunction, there is in principle no locality to any physical object. An object is only local or localized thru its scalar component or its mass.

Once we adopt this viewpoint, then everything, including what appears to be inexplicably instantaneous, or disjoint in time, becomes transparent to intelligentsia at the very least thru spectral analysis of superposition of states, the qualities and signatures of which having been well understood in the Quanto-Geometric order. Only because we have not understood the real properties of the quantum wavefunction, have we inferred a seemingly "spooky" character to it from the putative reductionist approach of statistical analysis and a supposed interpretative incompleteness of the latter. Every object's wavefunction is universally ubiquitous because it is ported or deployed within universal background space. An additional important fact is that mutual influence of wavefunctions does not propagate but enjoys in situ intermediacy.

\section{Entangled Particles as Surrogates of the Quan- to-Geometric Operator}

Quantum entanglement in a dynamic system of two particles is at first glance unmistakably a surrogation of the Qanto-Geometric ontological signature whereby one particle plays the role of the scalar tenet and the other the role of the space wavefunction tenet. What the two particles are doing is specifically emulating the state of the Quanto-Geometric object $\left\langle q_{4} \mid s_{4}\right\rangle$. The two particles, which at first glance form a phenomenological dynamic system protagonized by two distinct entities in travel, are in fact executing a constraint or mandate that imposes on them the behavior of a singular object. That is the reason and the only reason why they adopt the singlet state ontology well known to them. The two particles have become a single Quanto-Geometric object with themselves as the common ontological duet that is further engaged in mutual covariance per the Quanto-Geometric norm.

It is thru Quanto-Geometric ontological signature that they inter-relate with each other, causing what affects one te- net whether thru the wavefunction or the mass to resonate or entail consequences for the duet as a single object.

\section{Quantum Entanglement as the State of the Quanto-Geometric Quadratic (Radical) Opera- tor}

Let us recognize that the object or phenomenology that we are dealing with here is the Quadratic (Radical) Operator, reported in [1] [3], whose expression is:

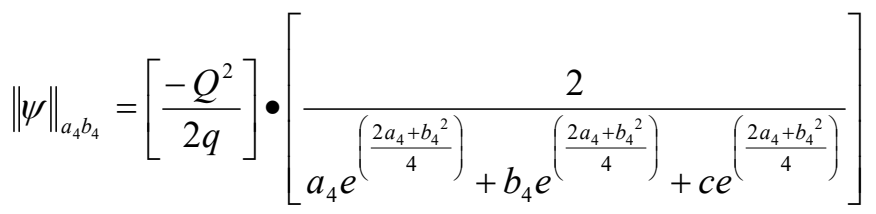

For this Operator:

$$
n=4, \mathrm{~s}_{4}= \pm \sigma
$$

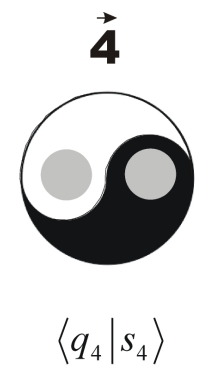

We refer the reader to [1] [2] for an explanation of the paramaters $a_{4}, b_{4}$ and $c$. In considering the properties of this Operator, it becomes outwardly clear that all ontology and phenomenology attributable to quantum entanglement belongs to the Quadratic Quanto-Geometric order, whereby the two basis ontological tenets are of equal "weight" and exert equated covariant influence on one another. Equal-weight covariant influence drives them to "seemingly" morph into one another, causing them to become indistinguishable from one another, albeit remaining each their own. If there was ever a point where the principle of mass-energy equivalence held true, it is manifestly the quanto-geometric quadratic covariant level. However any inquest in the principle of mass-energy equivalence as a possible interpretation of quantum entanglement is seldom realized because the real properties of the quantum wavefunction have remained unknown.

\section{Replicate of Quanto-Geometric Orthogonality in Quantum Entanglement}

The foremost postulate of Quanto-Geometric Theory is the existence of two tenets that cannot be defined in and of themselves, scalar and nothingness of space, that is, but only in relation to one another and specifically as opposite to or 
inverse of one another: one is everything that the other is not. This stands for the primordial condition of orthogonality [1] [2] as previously stated.

It is unquestionably the orthogonality characteristic of the Quanto-Geometric duet that is reproduced on the conjugate condition imposed on quantum entanglement state. Because quantum entanglement is expressly executed under the umbrella of the Quanto-Geometric ontological signature, do we see that the orthogonality feature finds its way thru the phenomenon with the mandate that the particles show a conjugate feature of some kind with respect to one another. Two bound electrons, the most ubiquitous form of quantum entanglement, are two twin elements with equal weight but with opposite spin (one up, one down). When it comes to photon particles for their own, for two photons of light to experience an entangled state as shown experimentally, they must have been spawn off two orthogonally polarized light beams, or otherwise subject to reversal of their polarization plane with respect to one another. Absent this conjugation character, entanglement cannot occur.

Conjugation in the entangled state is undoubtedly a replication of the anti-symmetric or orthogonal condition inherent to the Quanto-Geometric ontological signature.

\section{Replication of Quanto-Geometric Time-free covariance in Quantum Entanglement}

We have well explained at the beginning of this exposé why this theoretical framework is one of timelessness insofar as dynamic systems, and in particular that the covariant influence exerted on one another by the ontological tenets does not propagate in time but is instantaneously exerted.

Well, most unfathomable of all things, the covariant influence between two quantum entangled objects is an instantaneous observable. Notably, if the wavefunction of one particle is forced to collapse, the wavefunction of the other ipso facto follows suit. If one cares to understand that the environmental factor or laboratory setup that imposes the entangling constraints on the particles has in fact caused them to become a new object of Quanto-Geometric signature, one then readily gains the coveted insight into this mysterious behavior.

The question of how long it takes one particle to propagate its influence onto the other is no more sensible than the question of how long it took the electron to jump to the new value of momentum once the applied potential energy has shifted its quantum-wavefunction phase. Those effects are simply instantaneous. Once one understands that the two- particle dynamic system is in fact a new object quantogeometrically bound, independent of distance between the two particles which may be the infinites, then there is no more time problem or question.

Supplemental cause of the above is that space is totalitarian, allowing us to visualize the spatial component of the two objects surrogating the Quanto-Geometric condition to be:

- Very coherently in superposition with one another in their separation without vertical or horizontal bounds.

- Intimately and thoroughly merged with one another due to the symmetry in the properties of their wavefunction.

- That they are one with the tapestry of universal background space in the theater Shell of their interaction.

It is this totalitarian property of space that sustains the infinite development of harmonic waveforms at one single point or region of space as captured in more familiar Fourier analysis. If one takes the time to represent physically the possibility that a multitude of electric waveforms in even or odd harmonics can add up at one point, or how one point may represent both a positive peak and a negative peak or valley or even a node all at the same time, then one is forced to conclude that this wave or oscillation point, if it must be physical and not only representational, ought to be ultimately a spatial point and that space must be totalitarian (infinite foliate sum) in its tapestry both orthogonally and transversally.

Time is definitely not a part of this vision. That is what makes it possible with Laplace transforms to leave the time domain for achieving better solutions and representations. Ironically enough, in the Laplacian treatment we believe that the space domain is abstract and the time domain to be the real one! Once one has risen to the visualization provisioned by Fourier and Laplace, one should have the good sense and above all the courage to depose the mental model of time and not invoke the absurd of retro-causality for an interpretation, let alone seek to normalize it.

I will conclude this section by stating that it is every physicist's prerogative to put fancy math, clever math, copious math or perhaps obscure math behind any conundrum they face, but it can never substitute intelligentsia first. Retrocausation Theories, which have had a long insidious life ever since the birth of the Quantum Theory, shall all henceforth be disbanded and obliviated. 


\section{Quantum Entanglement in the Macrocosm}

A USA press story in early days of this millennium is of note when showing that entanglement exists not only in the quantum world but in the macroscopic world as well, in particular in the biosphere. It related an episode in the lives of two female twins who lived in the same city but in different sections of town. They visited each other as frequently as any pair of siblings did. When Christmas of that year came along, they met at one of their homes and exchanged gifts for the occasion. Holy surprise! They bought each other the exact same gift! To the reader who needs an explanation, just two words: Quantum Twins!

Is this example any different from the EPR photons at play in the midst of our laboratories? No, it is quantum entanglement or quantum phenomenon in our big macroscopic habitat. Note that the EPR photon experiment is seldom considered when looking for clues into Unification, a pursuit of long in our science, due to our deep-seated misunderstanding of the scales or Shells of matter. There are at least four world spans of a difference (or four Shells of matter) between two entangled electrons in an atomic orbital and two entangled photons in the space of our human laboratories. The EPR entangled photons are certainly an example of quantum entanglement or quantum phenomenon in the macrocosm that in itself could set the stage for reflections leading to the discovery of the true dimension of Unification. Sadly, it never has.

To express numerically the ontology of the new internally entangled singlet object $\left\|\psi_{44^{\prime}}\right\|$, or the entangled dynamic system as one pleases to name it, is to formulate a product between the wavefunction of one element and the scalar term of the other element respectively, all inserted in one bra-ket as denoted below:

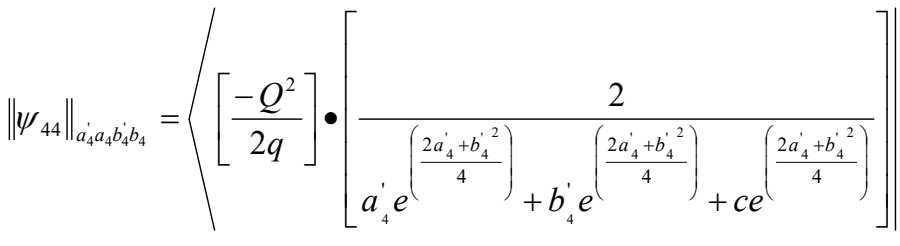

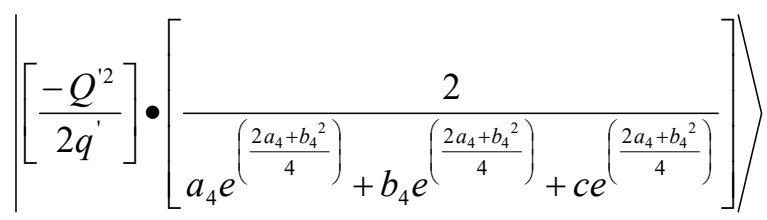

where the elements with the prime symbol represent those pertaining to the second twin. Since we have already ex- plained in [1] [2] how to approach the resolution of practical dynamic problems with the application of Quanto-Geometric operators, another repeat is counter-indicated here. I shall however put here in particular highlight that:

- the component objects do not have to be Quadratic objects but only the resulting singlet object.

- their standard deviation parameters in their quantogeometric expressions must be equal as a sine qua non condition.

If the component objects are quanto-geometric quadratic objects, then the resulting singlet object will become a superentangled object, by further potentializing the equivalency signature within the singlet and making the two subcomponents even more indistinguishable from one another.

\section{A Different View on Schrodinger's Cat}

The puzzle of all-time Quantum Physics! Let's frame it in E. Schrodinger's own words:

A cat is penned up in a steel chamber, along with the following device (which must be secured against direct interference by the cat): in a Geiger counter, there is a tiny bit of radioactive substance, so small, that perhaps in the course of the hour one of the atoms decays, but also, with equal probability, perhaps none; if it happens, the counter tube discharges and through a relay releases a hammer that shatters a small flask of hydrocyanic acid. If one has left this entire system to itself for an hour, one would say that the cat still lives if meanwhile no atom has decayed. The first atomic decay would have poisoned it. The $\psi$ function of the entire system would express this by having in it the living and dead cat (pardon the expression) mixed or smeared out in equal parts.

Physicists believe that the application of standard microscopic quantum mechanics to macroscopic objects, such as cats and computers require that the macroscopic objects do not always have unique classical state descriptions, but a series of superposed states that each may bear resemblance with a particular classical state. This interpretation of quantum dynamics begs the question of when it is that a quantum system stops existing as a superposition of states and becomes one or the other. It is clear that the cat, if he survives, will only remember the many episodes of his fleeting life, he will not remember having been dead or having undergone a state of death. The thought experiment vividly illustrates this apparent paradox. The experiment ties the fate of the cat to a quantum system that operates based on mixtures of states and directly transfers the quantum determinants and outcome to the macroscopic 
living entity, thereby requiring that the cat be both dead and alive at the same time before he reaches a final state, whether that state is a singlet one or even a multiplet or mixed state.

Because this paradox has proven to be unsolvable in all logics, physicists have principally relied on invoking artifacts of observation for its resolution. First critic of that line of interpretation, no other than legendary A. Einstein:

"Nobody really doubts that the presence or absence of the cat is something independent of the act of observation."

They contend that the act of observation decides what is real or determines which final state becomes real by collapsing the mixed wavefunction, because the cat cannot be dead and alive at the same time in any real outcome. The entangled wavefunctions here are: non-decayed nucleus - cat alive and decayed nucleus - dead cat. Therefore all the interpretations thus far offered to resolve this conundrum bear on observation and consequential collapse of the wavefunction. None has gained universal acceptation however, most likely on accounts of every one's realization that no aspect of physical reality can be dependent for its objective veracity and fullness on the presence, absence or action of a subjective and passive or perhaps inexistent third party or observer. Logically, the cat will either be dead or remain alive whether or not some curious human has opened the door to take stock of the outcome.

From the interpretative standpoint, the problem with this thought experiment is that it is based on mistaken assumptions and a misunderstanding of the physics of life. Most significantly, it is erected on the ignorance of the most fundamental axiom of physical law: Quanto-Geometry. What one firstly ought to understand is that life is both a condition of vitality and a condition of death. There is not a single human being that would live perhaps an ephemeral second if the army of cells that have become deficient during homeostasis are not in continual succumb to the process of death instigated by their lysosomes, in addition to those succumbing by apoptotic control, a genetic program orchestrating cell death throughout the entire anatomy of a living body as a necessary condition to support homeostasis. Out of the 37 trillion cells or so of an adult human body an average of 25 billion of them die every day under apoptotic mandate. So therefore the condition of vitalization rests on the ensemble of living cells in our body which promote and sustain the meta-level histological blocks and processes, while the condition of death rests on the ensemble of dead cells and the systems devoted to the orchestration of death in our body.

From a Quanto-Geometric viewpoint, the condition of vitalization plays the role or function of the spatial wavefunc- tion tenet, because its north is motion and expansion. Whereas the condition of death plays the role of the static scalar, because in death, as demonstrated in a dead body, there is only inactivity and staticity as the ultimate pursuit and realization. The Quanto-Geometric condition of orthogonality is so very explicitly present in life and death as a duet. Are they not completely anti-symmetric to one another? Therefore every living entity is both alive and dead at the same time, in accordance with the Quanto-Geometric ontological signature.

There is no possible way to rationalize the human experience in a life traversal without assuming an orchestrator of pro-pensive or pro-life processes and another orchestrator of death or shadow processes, all based on twin ontology in every human. On that account, every human is one or one persona in active life under the sun, and another persona in inactive or quasi static life in the silence and quiet of our nights. It is not without reason that our bodies all mimic death during night sleep. At every instance of life a human being is this perfect quantum twin in every which way it is defined. A life traversal is the story of a quanto-geometric covariant duet spanning the 9 normalized phases of ontological development. One aspect of the shadow persona is indeed instructed in the psychoanalytic school of psychology, under the denomination of shadow personality or alter ego.

The fact remains that the wavefunction of the orchestrator of life and the wavefunction of the prostrated alter ego are both present in the individual's body, whose life course is the entire suite of Quanto-Geometric normalized covariant signatures. According to this view, at birth there is death and at death there is vibrancy of life, to E. Schrodinger's posthumous complete satisfaction despite his incredulity, as he wrote:

The $\psi$ function of the entire system would express this by having in it the living and dead cat (pardon the expression) mixed or smeared out in equal parts.

Except of course that the dead cat is not visibly smeared out but that its dead parts pertain to the internal and quasi unobservable province of the alter ego orchestrator. While it might be difficult for the rational mind of a physicist to comprehend the origin of death at birth in its complete orchestration (unless one calls for the trans-physical dimensions of the Omniverse and their metrics), it is fairly simple to represent presence of life at death as embodied in the known process of agony. The better part of agony is a lethargic state similar to hibernation where the distinction between life and death vanishes because they have punctiliously merged into one another. Furthermore, anyone, unafraid enough to study in detail the development of necrological processes in the prostration of death, will know that the many body's histological systems 
never shut down all at once, the sense of audition for instance always persisting to the very end across the histological blocks that make it possible. On that account alone, in the state of prostration of death, the body goes through a transition of mixed states conformed in low-life systems/dead systems, until all histological systems have absolutely shutdown (at an advanced point in the transcendental phase).

Given all of the above, the manicheic view that the living must be either alive or dead, and that two stateful wavefunctions representing both conditions in one single individual at any one time cannot reasonably be claimed, simply stand for a mischaracterization. Schrodinger's cat, akin to all living entities of the biosphere, is indeed both alive and dead at any one moment, independent of all forms of observation. Collapsing the wavefunction of the prostrated twin will indeed collapse the entire ontology. However, third party observation does not entail such effects.

\section{Conclusion}

What we have accomplished in this thesis is a clear and detailed exposition, we hope, of the foundational descriptors of motion in dynamic systems thru an entirely timeindependent kinematic regime. We have presented the atemporal defoliation rate of the spatial vacuum as the central element at the heart of the evolution of dynamic systems. We have uncovered the panoply of primitives of motion responsible at first order for momenta thru the eigenkinetics and eigenforms essentially deriving from the Quanto-Geometric 2-space metric. Lastly, we have de-ciphered the obscure and cryptic state code behind the dynamics of quantum entanglement phenomena. These elements altogether constitute the root methodology for a granular description of any type of motion in dynamic systems in the timeless regime.

Shell symmetry, based on the referred 2-space metric, emerges as a fact of paramount significance when analyzing stateful behavior, generally attributed to objects, with respect to dynamic behavior, generally attributed to multi-partite systems, for a better comprehension of the both in their natural courses. Both quantum entanglement and the experimental quantization of free travel electrons prove without a doubt that the evolution of all dynamic systems may be properly understood in terms of stateful behavior of objects in all Shells of matter under the most fundamental of physical laws: QuantoGeometric scalar-space covariance.

In closing this study, we open two windows onto the future of quantum physics in terms of two predictions projected from the implications of this study:
- Because all phenomenologies occur thru the many de-ploys of space, which leaves nothing in a nonexistent fleece of behind or arrearage, nor in time which is a fleece of fiction, everything that ever occurred must still be existent or preserved within the tensored deploys of space for perennial direct dimensional observation or re-enactment, in the most concrete materialization of dimensional atemporality or perpetual present.

- Because space is totalitarian and with no absolute front end to its omniversal deployment, there must exists a spatial force, of which imagination of the living is only an incipient aspect, by which all projected reality thru a sufficient degree of that force is conformally driven to existential materialization, without loss of homogeneity in the continual space deploy and spatial tapestry of the Omniverse.

\section{REFERENCES}

[1] Jean-Claude, J. Joseph, Quanto-Geometry: Overture of Cosmic Consciousness and Universal Knowledge for All, Volume I, Quantometrix, 2015

[2] Jean-Claude, J. Joseph, Quanto-Geometric Tensors \& Operators on Unified Quantum-Relativistic Background, Academia.edu, November 2016 - Research Gate, 2017

[3] Jean-Claude, J. Joseph, Quanto-Geometry: Overture of Cosmic Consciousness and Universal Knowledge for All, Volume II, work in progress

[4] Jean-Claude, J. Joseph, A Final Cure to the Tribulations of the Vacuum in the Quantum Theory, ResearchGate, April 17, 2017

[5] ACOSTA, V., and COWAN, C., and GRAHAM, B.J., Essentials of Modern Physics, Harper \& Row Publishers, 1973

[6] Cruz, Andoni, Chamizo, Jose, Garritz, Diana, Estructura Atómica: Un Enfoque Químico, Fondo Educativo Interamericano, 1986

[7] Robert L. Oldershaw, Towards A Resolution Of The Vacuum Energy Density Crisis, arXiv:0901.3381, 2009

[8] Asher Peres, Critique of the Wheeler-Dewitt Equation, arXiv:gr-qc/9704061, 1997

[9] John C. Baez, Emory F. Bunn, The Meaning of Einstein's Equation, E.F.B., 2006 
[10] Young, Nicholas, An Introduction to Hilbert Space, Cambridge University Press, 1988

[11] D. Halliday, R.Resnick, J. Walker, Fundamentals of Physics, $5^{\text {th }}$ Edition, John Wiley \& Sons, 1997

[12] Giovanni Sansone (translated by Ainsley H. Diamond), Orthogonal Functions, Interscience Publishers, 1959

[13] Courant, R.; Hilbert, D. Methods of Mathematical Physics

[14] Yitzhak Katznelson, An Introduction to Harmonic Analysis, $3^{\text {rd }}$ Edition. Cambridge University Press, 2004

[15] Sunder, V.S. Functional Analysis: Spectral Theory, Birkhäuser Verlag, 1997

[16] Farwaz T. Ulabi, Michel M. Maharbiz, Circuits, National Technology and Science Press, 2009

[17] Weber L. Robert, Manning V. Kenneth, White Marsh W, Physique Générale, McGraw-Hill Canada, 1967

[18] Jean-Claude, J. Joseph, The Mirage of Time Throughout Modern Physics: Scandalous Life of an Impostor, Research Gate, 2017 